\title{
Low-Cost Quasi-Distributed Sensor Based on Bended Side- Polished Plastic Optical Fiber
}

\author{
M. Lomer, C. Galíndez, J. Zubía ${ }^{1}$, A. Quintela, F. Madruga, J. M. López Higuera \\ Photonics Engineering Group - Universidad de Cantabria \\ Av. De Los Castros s/n, Santander E39005, SPAIN \\ Phone: +34-942-201495 Fax: +34-942-201873 \\ Email:lomer@teisa.unican.es \\ ${ }^{1}$ ETSI Bilbao Universidad del Pais Vasco (Spain) jtpzuzai@bi.ehu.es.
}

\begin{abstract}
A novel quasi-distributed sensor based on transmittance changes induced by core fiber sidepolished on a bent optical fiber is shown. The measurement points are distributed over a loop of $\mathrm{N}$ fibers full-turned. The prototype and experimental results are presented.

(C)2006 Optical Society of America

OCIS codes (060.2370) General; (060.2280) General
\end{abstract}

\section{Introduction}

Adequate control systems and physical or chemical parameters always represents a common concern for the industry. In the last years, optical fiber sensors have contributed with different new solutions; where monomode and multimode glass fibers have been used as part of the sensors, these sort of systems are well appreciate in metrology, mainly the low cost ones [1]. New advances in the fabrication of plastic optical fibers (POF) have contributed to expand their application fields; and they also represent a low cost solution [2-4]. In fact, using POF offer several advantages, like low weight, flexibility, easy handling, large core diameter and large numerical aperture. Actually, POF with diameters between 0.125 and $3 \mathrm{~mm}$, and typical transmission losses of $120 \mathrm{~dB} / \mathrm{Km}$ are commercially available.

Several liquid level sensors made with optical fibers have been reported previously, they use diverse techniques [5-7]; some of them take the measure in just one point, whereas others are so sophisticated. In this document we present a new quasi-distributed sensor based on lateral-polished of POF bends. This lateral polished generates an elliptical surface, where a part of the core has been removed. The measurement points are laterally distributed along the fiber, placed over each loop of the $\mathrm{N}$ fiber full-turns. A change in the refraction index of the medium in contact with the core produces variations on the transmittance. These changes are well detected in the output fiber ending. The lateral polished produce moderated losses, being possible measure with numerous points along the entire fiber. In the next sections we present a sensor design and the experimental results obtain with the prototype developed.

\section{Transducer concept}

The transducer architecture is integrated by $\mathrm{N}$ loops of a proper POF. Each fiber loop is side-polished as shown in the figure 1 . When the side-polished area of each loop is covered by a liquid, the loop transmission losses change; hence the presence of liquid is detected. As consequence of the $\mathrm{N}$ fiber side-polished loops disposed over a cylindrical structure, the quasi-distributed transducers (providing $\mathrm{N}$ measurement points on a single fiber) can be used in the design of liquid level sensor systems. Distances between measurement points range and resolutions are determined by the characteristics of the fiber and loops size.

\section{Transducer design}

In Figure 1, a full-turned side polished fiber scheme is shown. This design considers a typical fiber POF with a diameter of $2 \mathrm{~mm}$ including the jacket. The core diameter (2a), cover diameter (2b) and jacket diameter (2c) have $0.98,1.0$ y $2.0 \mathrm{~mm}$ respectively. Side polish on the bent region forms an elliptical surface where the short and long axis have diameters of $2 \mathrm{x}$ and $2 \mathrm{y}$ respectively. The maximum thickness of the core removed by polishing is calculated by using a simple geometrical equation, $\varepsilon=(a+R)-\left[(a+R)^{2}-y^{2}\right]^{1 / 2}$, where $a$ and $R$ are the core and bend radius. They value is obtained by measuring the long axis of the ellipse. Conditions for propagation of light in this wave-guided model can be analyzed simply by approaching a plane waveguide. In fact, several meridian rays 
entering the curved region do not accomplish the conditions of total internal reflection when they have incidence in the core-cover interface. Therefore, some rays will be partially reflected and the others will be radiated out. The last one represents losses for bend. On the other hand, in addition to bend losses, incident rays to the polished area can have losses because they find a modified surface and also the core-cladding interfaces have been replaced by an external medium-core. Total losses depend on the opto-geometric parameters of the fiber, the radius of bend $\mathrm{R}$ and the thickness of the polished core $\varepsilon$. Consequently, changes of losses on the polished surface interfaces are used in the sensor performance.

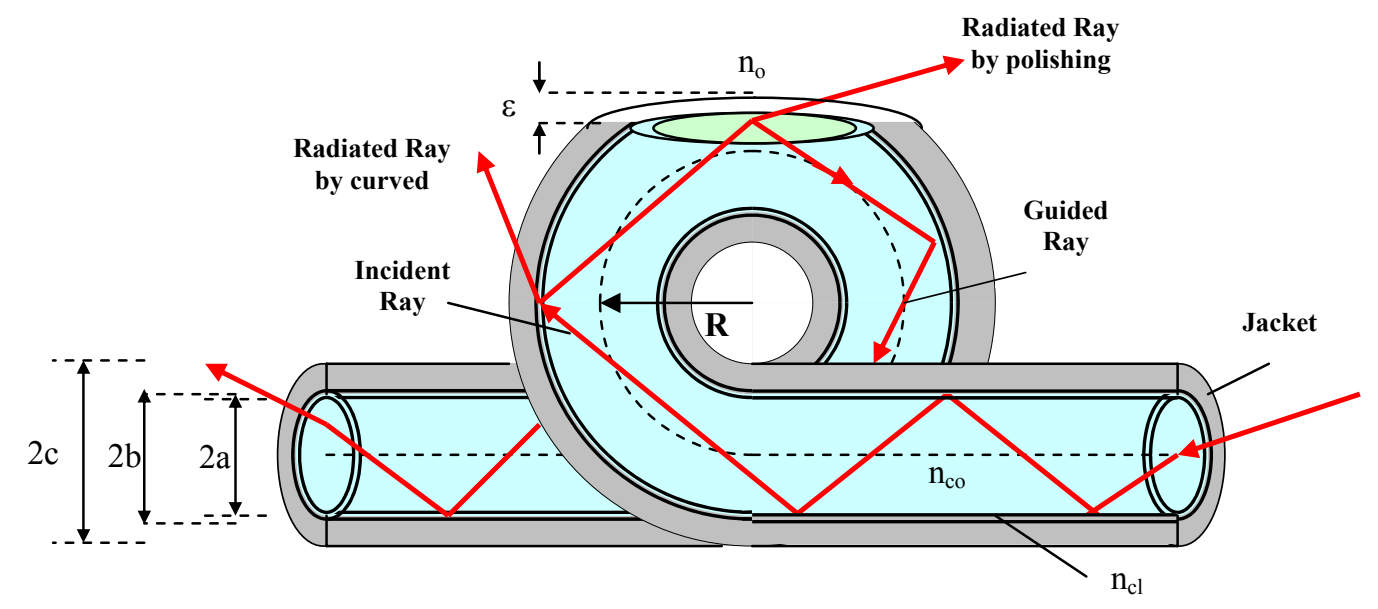

Fig.1. Illustration of one POF side-polished loop of the quasi-distributed transducer.

The spiral of the fiber that has a constant section is characterized by the radius of bend of a full-turn spiral R', in function of pitch $\mathrm{P}$ and the fiber radius of bend, which is given by:

$$
\mathrm{R}^{\prime}=\mathrm{R}+(1 / \mathrm{R})[\mathrm{P} /(2 \pi)]^{2}
$$

And the fiber length per period

$$
\mathrm{L}=\left[(2 \pi \mathrm{R})^{2}+\mathrm{P}^{2}\right]^{1 / 2}
$$

Radius R y R' are related to the fiber axis. R' and L represent the distances for measurement and the fiber length required for the sensor in each point of measurement. By using POF of diameter $2 \mathrm{~mm}$, including the jacket and $\mathrm{R}=$ $5 \mathrm{~mm}$, the values obtained for $\mathrm{R}^{\prime}$ and $\mathrm{L}$ are $\mathrm{R}^{\prime}=5.02 \mathrm{~mm}$ and $\mathrm{L}=31.5 \mathrm{~mm}$.

In Figure $2 \mathrm{a}$ characteristics for bend losses and polish of a full-turn of fiber are shown, considering a radius $\mathrm{R}=5 \mathrm{~mm}$. The fiber used is a PMMA-POF index step with diameter $2 \mathrm{~mm}$ including the jacket, where core index and cover index are $n_{c o}=1,492$ and $n_{c l}=1,402$ respectively. A source of light LED in wavelength of $660 \mathrm{~nm}$ has been used. Direction of polishing goes from the external interface of the jacket toward the center of the fiber axis (Fig. 2b). In the absence of polishing and with a total fiber length of two meters, the contribution to loss was observed to be $4.0 \mathrm{~dB}$. However, theoretical calculations predict more than $4.5 \mathrm{~dB}$ of loss. The difference is due to the finite cladding thickness, of only $10 \mu \mathrm{m}$, which enables part of the light radiated to the cladding to be reflected towards the core again after having reached an outer interface. As a matter of fact, a very thin layer of air is present at the cladding-jacket interface, which facilitates reflections and may act as a waveguide as well. In the experimental setup, the polishing depth $\varepsilon$ was measured by means of an optical microscope, with an accuracy of $\pm 10 \mu$ m. It should be noted that loss remains constant until the polishing penetrates the core. In such a case, loss increases linearly. Every step of $100 \mu \mathrm{m}$ the increase is approximately $3 \mathrm{~dB}$. For $\varepsilon \approx 400 \mu \mathrm{m}$, loss is $13 \mathrm{~dB}$ higher than for $\varepsilon=0$. In sensing applications it can be enough a thickness of $\varepsilon=0.1 \mu \mathrm{m}$ that represents an increment of $1 \approx \mathrm{dB}$ of additional losses to the losses for bend for a $\mathrm{R}=5 \mathrm{~mm}$. The election of a radius of weak bend is justified because sensibility increases with the external environment 


\section{TuE49.pdf}

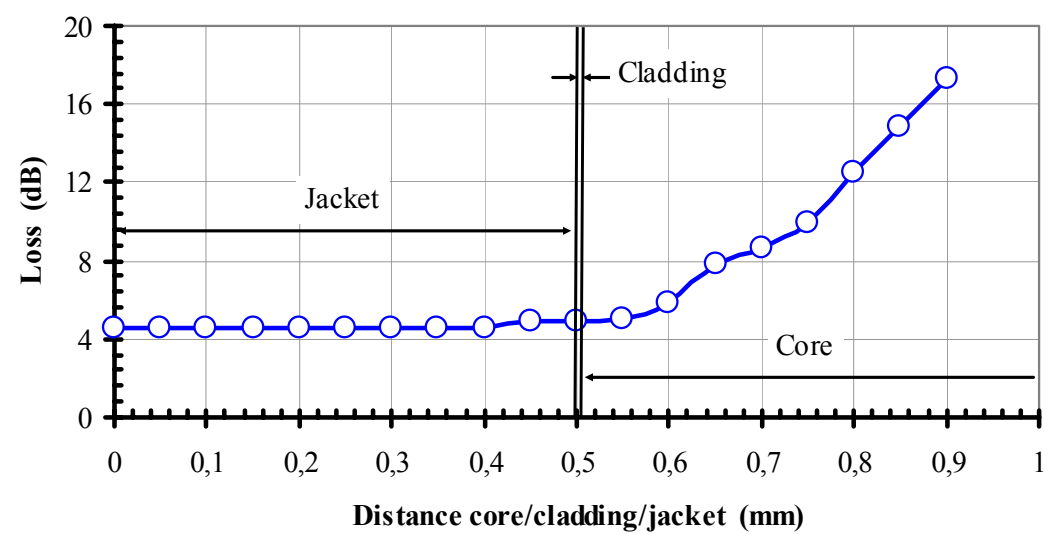

(a)

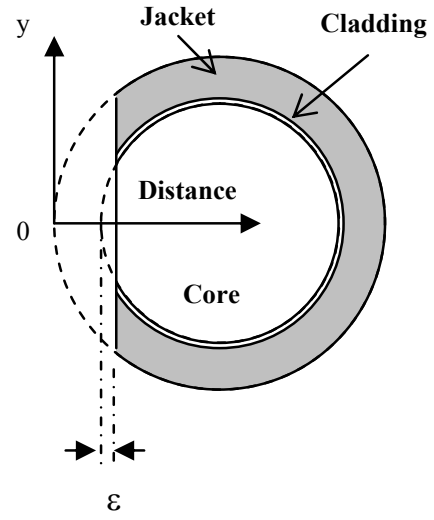

(b)

Fig.2. a) Evolution of bend losses of one POF side polished lop of $\mathrm{R}=5 \mathrm{~mm}$. b) Polish sense in a straight section of the curved fiber

\section{Transducer fabrication and experimental results}

The sensor prototype is with spirals of fiber over a cylindrical rod with constant cross section and radius $\mathrm{R}=5 \mathrm{~mm}$. The lateral polished of each fiber spiral is made in the cylindrical axis sense. The maximum removed thickness from the core is $0.1 \mathrm{~mm}(2 \mathrm{y}=2 \mathrm{~mm})$ that corresponds to additional losses of $1 \mathrm{~dB}$ for each fiber spiral. In Fig. 3 photos of sensor set up and details are sown. The device is placed vertically with respect to the tank bottom, initially empty. In Fig. 4 experimental results for 15 points of measurements, when the tank is filled with water are shown. A He-Ne at $632.8 \mathrm{~nm}$ laser has been used as light source. The separation point is $2.2 \mathrm{~mm}$ (pitch P). The total losses due to bending fibers and the polished are $45.3 \mathrm{~dB}$. When water starts to fill the tank, the liquid level is increased and polished spirals are covered, these register variations in the transmittance. Replacing the surrounded media, initially air by water induces a change in the losses of an amount of $0.16 \mathrm{~dB}$. This variation is enough to be sensed by a photodiode PIN placed in the fiber ending. The precision on the measure is given by the ellipse minor axis diameter formed by the initial polished $(2 \mathrm{x}=0.08 \mathrm{~mm})$.

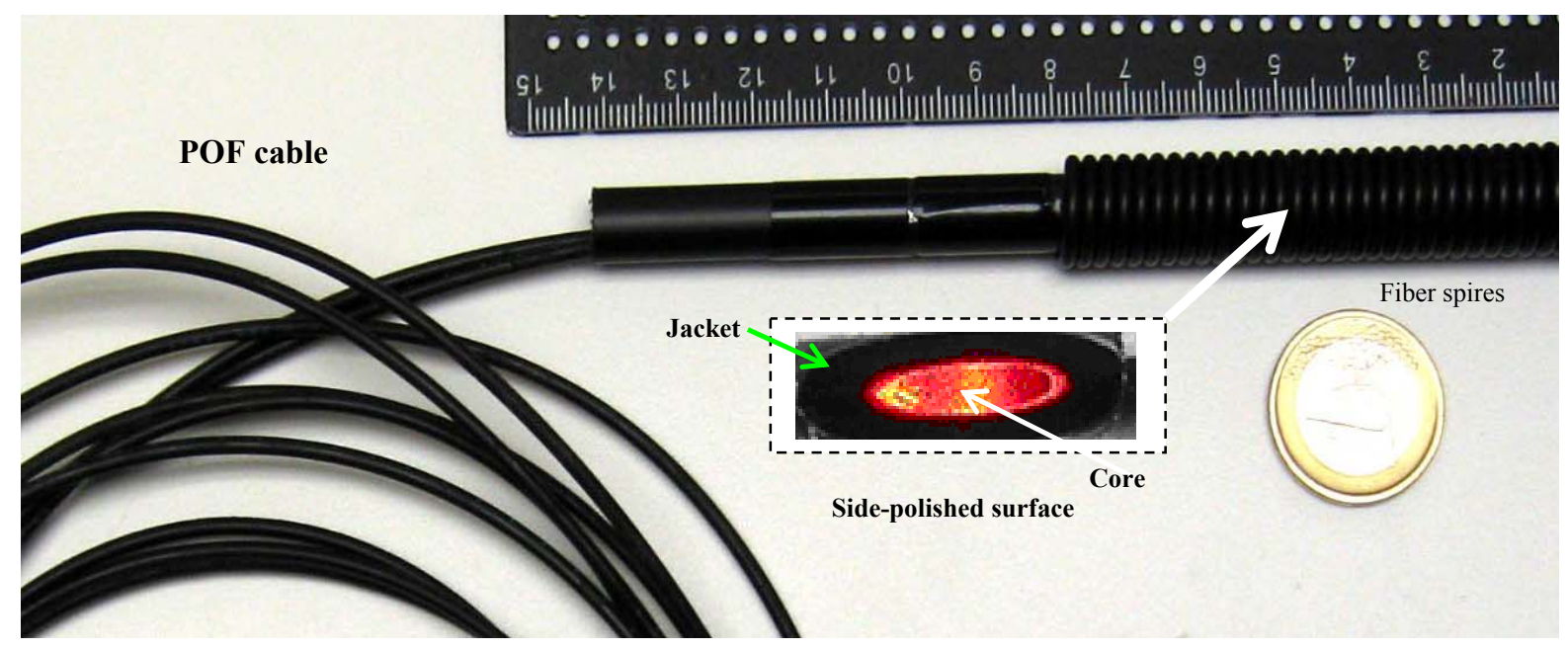

Fig. 3. Transducer view with insets to illustrate the optical light radiation of one side polished loop. To compare the size a coin of 1 euro is placed. 
The results in Figure 4 show that losses decrease when the polished surfaces have contact with water. Apparently, this result would be contradictory to theory. But it can be explained by the use of POF cable including jacket. In fact, there is an air gap between the jacket and optical cladding of the fiber. As an effect of the bending, the rays radiated outside the fiber, are propagated in this gap going out (to the exterior) through the polished region of the fiber. On the other hand, when the polished area is covered by water, an interface air-water appears. Therefore, some incidence rays are reflected toward the interior of the core.

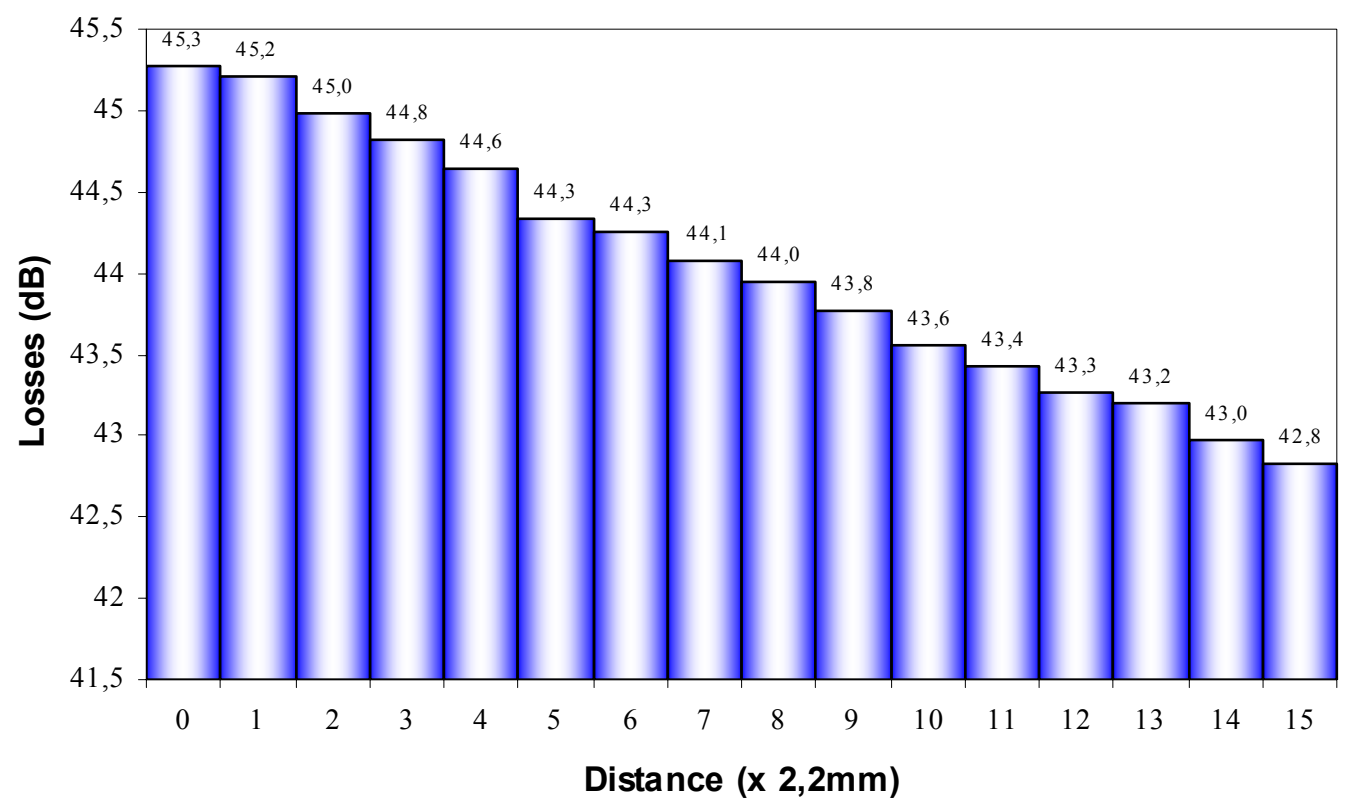

Fig. 4. Experimental loss obtained as a function of distance of immersed in water.

\section{Conclusions}

The paper presents a very simple new quasi-distributed optical fiber sensor. It is based on changes of transmittance, when a liquid replaces the surrounding air over the lateral-polished surface of a bended optical fiber. The direct contact between the core and the medium under test provides an important punctual sensibility. It has been demonstrated the functioning of a quasi-distributed measurement system along $33 \mathrm{~mm}$ length with 15 points of measured. Due to the flexibility of plastic optical fiber it is possible to have a wide measurement range, from millimeters to several meters of length. The introduced advantages by the POF make possible develop very low cost sensing systems.

\section{Acknowledgments}

The authors acknowledge the Spanish Government because; this work has been developed under the research projects TEC200405936-C02-02 and TEC2005-08218-CO2.

\section{References}

[1] J.M.Lopez-Higuera Handbook of optical fiber sensing technology (John Wiley and Sons Ltd., England 2002)

[2] R.J. Bartlett, R.P. Chandy, P. Eldridge, D.F. Merchant, R.Morgan and P.J. Scully, "Plastic optical fibre sensor and devices", Trans. Inst. Measurement and Control, 22, 431-457,(2000).

[3] M. Tekelloglu and B.D. Wood, "Prediction of light-transmission losses in plastic optical fibers", App. Opt., 44, 2318-2326, (2005).

[4] P. Polishuk, "Plastic optical fiber builds on MOST success", Laser Focus World, 42, 57-61, March 2006.

[5] J.P. Dakin and M.G. Holliday, “A liquid level sensor based on O-H or C-H absorption monitoring”, Proceeding of the First International Conference on Optical Fibre Sensor, (Institution of Electrical Engineering, London, 1983), pp. 91-95.

[6] D.A. Jackson, "High precision remote liquid level measurement using a combination of optical radar and optical fibres", Proceeding of the First International Conference on Optical Fibre Sensor, (Institution of Electrical Engineering, London, 1983), pp. 100-103.

[7] D. Donlagic and B. Culshaw, "Microbend sensor structure for use in distributed and quasi-distributed sensor systems based on selective lauching and filtering of the modes in graded index multimode fiber", J. Lightwave Technol, 19, 1856-1868 (1999). 